\title{
THE ANALYTIC RADON-NIKODYM PROPERTY IN LEBESGUE BOCHNER FUNCTION SPACES
}

\author{
PATRICK N. DOWLING
}

\begin{abstract}
Let $X$ be a complex Banach space, $(\Omega, \Sigma, \mu)$ a finite measure space, and $1 \leq p<\infty$. Then $L_{p}(\mu ; X)$ has the analytic Radon-Nikodym property if and only if $X$ has it.
\end{abstract}

1. Introduction. In [2] Bukhvalov and Danilevich introduced the notion of the analytic Radon-Nikodym property. The relationship between the analytic RadonNikodym property and the existence of bounded holomorphic embeddings has been studied by Aurich [1], while its relationship with representable operators has been studied by Dowling [5]. Recently, Edgar [7] has shown that a complex Banach space has the analytic Radon-Nikodym property if and only if every $L_{1}$-bounded analytic martingale in $X$ converges. In this note we are going to show that if a complex Banach space $X$ has the analytic Radon-Nikodym property then so does $L_{p}(\mu ; X)$ for $1 \leq p<\infty$, where $(\Omega, \Sigma, \mu)$ is a finite measure space.

\section{Preliminaries.}

DEFINITION 1. A complex Banach space $X$ has the analytic Radon-Nikodym property if every $X$-valued measure of bounded variation, defined on the Borel subsets of $\mathbf{T}(=\{z \in \mathbf{C}:|z|=1\})$, whose negative Fourier coefficients vanish, has a Radon-Nikodym derivative with respect to normalized Lebesgue measure $d \theta / 2 \pi$ on $\mathbf{T}$.

DEFINITION 2. Let $X$ be a complex Banach space. The space $H^{p}(X)$ consists of all holomorphic functions $f: D \rightarrow X$, where $D$ is the open unit disc in $\mathbf{C}$, and satisfying $\|f\|_{p}<\infty$ where

$$
\begin{aligned}
\|f\|_{p} & =\sup _{0 \leq r<1}\left(\int_{0}^{2 \pi}\left\|f\left(r e^{i \theta}\right)\right\|^{p} \frac{d \theta}{2 \pi}\right)^{1 / p}, \quad 0<p<\infty \\
\|f\|_{\infty} & =\sup _{z \in D}\|f(z)\| .
\end{aligned}
$$

For details on vector-valued holomorphic functions see $[\mathbf{4}, \mathbf{8}] \cdot\left(H^{p}(X),\|\cdot\|_{p}\right)$ is a Banach space for $1 \leq p \leq \infty$.

THEOREM 1 [2]. Let $X$ be a complex Banach space. The following are equivalent:

(a) $X$ has the analytic Fadon-Nikodym property,

(b) for some $p, 1 \leq p \leq \infty$, every function in $H^{p}(X)$ has radial limits a.e.,

(c) for all $p, 1 \leq p \leq \infty$, every function in $H^{p}(X)$ has radial limits a.e.,

Received by the editors November 17, 1985.

1980 Mathematics Subject Classification (1985 Revision). Primary 46E30, 46G10; Secondary 46B22. 
(d) for some $p, 1 \leq p<\infty, \lim _{r \uparrow 1} f_{r}$ exists in $L_{p}(\mathbf{T} ; X)$ for every $f \in H^{p}(X)$, where $f_{r}\left(e^{i \theta}\right)=f\left(r e^{i \theta}\right)$,

(e) for all $p, 1 \leq p<\infty, \lim _{r \uparrow 1} f_{r}$ exists in $L_{p}(\mathbf{T} ; X)$ for every $f \in H^{p}(X)$.

3. The analytic Radon-Nikodym property for $L_{p}(\mu ; X)$.

THEOREM 2. Let $X$ be a complex Banach space, $1 \leq p<\infty$, and $(\Omega, \Sigma, \mu) a$ finite measure space. Then $L_{p}(\mu ; X)$ has the analytic Radon-Nikodym property if $X$ does.

PROOF. Let $f \in H^{p}\left(L_{p}(\mu ; X)\right)$. Then $f(z)=\sum_{n=0}^{\infty} a_{n} z^{n}$, where $a_{n} \in L_{p}(\mu ; X)$ for all $n \in \mathbf{N}$. Since $f$ is holomorphic we have that, for each $0<r<1$,

$$
\sum_{n=0}^{\infty}\left\|a_{n}\right\| r^{n}<\infty
$$

that is, for each $0<r<1$,

$$
\sum_{n=0}^{\infty}\left(\int_{\Omega}\left\|a_{n}(\omega)\right\|^{p} d \mu(\omega)\right)^{1 / p} r^{n}<\infty .
$$

Hence, for each $0<r<1$,

$$
\sum_{n=0}^{\infty}\left(\int_{\Omega}\left\|a_{n}(\omega)\right\|^{p} d \mu(\omega)\right) r^{n}<\infty,
$$

and so, for each $0<r<1$,

$$
\int_{\Omega}\left(\sum_{n=0}^{\infty}\left\|a_{n}(\omega)\right\|^{p} r^{n}\right) d \mu(\omega)<\infty .
$$

Now, for each $0<r<1$,

$$
\sum_{n=0}^{\infty}\left\|a_{n}(\omega)\right\|^{p} r^{n}<\infty
$$

for almost all $\omega \in \Omega$, and from this we get that, for each $0<r<1$,

$$
\sum_{n=0}^{\infty}\left\|a_{n}(\omega)\right\| r^{n}<\infty
$$

for almost all $\omega \in \Omega$. Thus, the function $f(\cdot)(\omega): D \rightarrow X$, defined by $f(z)(\omega)=$ $\sum_{n=0}^{\infty} a_{n}(\omega) z^{n}$, is holomorphic for almost all $\omega \in \Omega$.

$$
\infty>\|f\|_{p}^{p}=\sup _{0 \leq r<1} \int_{0}^{2 \pi}\left\|f\left(r e^{i \theta}\right)\right\|^{p} \frac{d \theta}{2 \pi}=\lim _{r \uparrow 1} \int_{0}^{2 \pi}\left\|f\left(r e^{i \theta}\right)\right\|^{p} \frac{d \theta}{2 \pi}
$$

(since $\|f(\cdot)\|$ is subharmonic)

$$
\begin{aligned}
& =\lim _{r \uparrow 1} \int_{0}^{2 \pi} \int_{\Omega}\left\|f\left(r e^{i \theta}\right)(\omega)\right\|^{p} d \mu(\omega) \frac{d \theta}{2 \pi} \\
& =\lim _{r \uparrow 1} \int_{\Omega} \int_{0}^{2 \pi}\left\|f\left(r e^{i \theta}\right)(\omega)\right\|^{p} \frac{d \theta}{2 \pi} d \mu(\omega) \\
& =\int_{\Omega}\left(\lim _{r \uparrow 1} \int_{0}^{2 \pi}\left\|f\left(r e^{i \theta}\right)(\omega)\right\|^{p} \frac{d \theta}{2 \pi}\right) d \mu(\omega)
\end{aligned}
$$


(by the Monotone convergence theorem)

$$
\begin{aligned}
& =\int_{\Omega}\left(\sup _{0 \leq r<1} \int_{0}^{2 \pi}\left\|f\left(r e^{i \theta}\right)(\omega)\right\|^{p} \frac{d \theta}{2 \pi}\right) d \mu(\omega) \\
& =\int_{\Omega}\|f(\cdot)(\omega)\|_{p}^{p} d \mu(\omega) .
\end{aligned}
$$

Therefore, for almost all $\omega \in \Omega,\|f(\cdot)(\omega)\|_{p}<\infty$; that is, for almost all $\omega \in \Omega$, $f(\cdot)(\omega) \in H^{p}(X)$. By Theorem 1 and the assumption that $X$ has the analytic Radon-Nikodym property we have that, for almost all $\omega \in \Omega, \lim _{r \uparrow 1} f_{r}(\cdot)(\omega)$ exists in $L_{p}(\mathbf{T} ; X)$. Moreover,

$$
\begin{aligned}
\left\|f_{r}-f_{s}\right\|_{L_{p}\left(\mathbf{T} ; L_{p}(\mu ; X)\right)}^{p} & =\int_{0}^{2 \pi}\left\|f\left(r e^{i \theta}\right)-f\left(s e^{i \theta}\right)\right\|^{p} \frac{d \theta}{2 \pi} \\
& =\int_{0}^{2 \pi} \int_{\Omega}\left\|f\left(r e^{i \theta}\right)(\omega)-f\left(s e^{i \theta}\right)(\omega)\right\|^{p} d \mu(\omega) \frac{d \theta}{2 \pi} \\
& =\int_{\Omega} \int_{0}^{2 \pi}\left\|f\left(r e^{i \theta}\right)(\omega)-f\left(s e^{i \theta}\right)(\omega)\right\|^{p} \frac{d \theta}{2 \pi} d \mu(\omega) \\
& =\int_{\Omega}\left(\left\|f_{r}(\cdot)(\omega)-f_{s}(\cdot)(\omega)\right\|_{L_{p}(\mathbf{T} ; X)}^{p}\right) d \mu(\omega) .
\end{aligned}
$$

But

$$
\left\|f_{r}(\cdot)(\omega)-f_{s}(\cdot)(\omega)\right\|_{L_{p}(\mathbf{T} ; X)} \rightarrow 0
$$

as $r, s \uparrow 1$ for almost all $\omega \in \Omega$ and all

$$
\left\|f_{r}(\cdot)(\omega)-f_{s}(\cdot)(\omega)\right\|_{L_{p}(\mathbf{T} ; X)} \leq 2\|f(\cdot)(\omega)\|_{p} .
$$

Hence by the dominated convergence theorem,

$$
\left\|f_{r}-f_{s}\right\|_{L_{p}\left(\mathbf{T} ; L_{p}(\mu ; X)\right)}^{p} \rightarrow 0
$$

as $r, s \uparrow 1$. Thus $\lim _{r \uparrow 1} f_{r}$ exists in $L_{p}\left(\mathbf{T} ; L_{p}(\mu ; X)\right)$ and so, by Theorem $1, L_{p}(\mu ; X)$ has the analytic Radon-Nikodym property.

REMARK. In [3] it is shown that $L_{p}(\mu ; X)$ has the Radon-Nikodym property if $X$ has it and if $1<p<\infty$. Theorem 2 is the analogue of this result but the method of proof is quite different.

ACKNOWLEDGEMENTS. This work was completed while the author was visiting University College, Dublin, Ireland. The author would like to thank Séan Dineen for many helpful discussions and suggestions during the preparation of this paper.

\section{REFERENCES}

1. V. Aurich, Bounded holomorphic embeddings of the unit disk into Banach spaces, Manuscripta Math. 45 (1983), 61-68.

2. A. V. Bukhvalov and A. A. Danilevich, Boundary properties of analytic and harmonic functions with values in Banach spaces, Math. Notes 31 (1982), 104-110.

3. J. Diestel and J. J. Uhl, Vector measures, Math. Surveys, no. 15, Amer. Math. Soc., Providence, R.I., 1977.

4. S. Dineen, Complex analysis in locally convex spaces, North-Holland Math. Stud., vol. 57, NorthHolland, Amsterdam, 1981. 
5. P. N. Dowling, Representable operators and the analytic Radon-Nikodym property in Banach spaces (to appear).

6. G. A. Edgar, Complex Martingale convergence, preprint.

7. __ Analytic martingale convergence, preprint.

8. E. Hille and R. S. Phillips, Functional analysis and semi-groups, Amer. Math. Soc. Colloq. Publ., vol. 31, Amer. Math. Soc., Providence, R. I., 1957.

Department of Mathematical Sciences, Kent State University, Kent, Ohio 44242

Current address: Department of Mathematics, Ohio State University, Columbus, Ohio 43210 